J. Clin. Chem. Clin. Biochem.

Vol. 21, 1983, pp. 381-386

\title{
Cholinesterase (EC 3.1.1.8) mit Butyrylthiocholin-iodid als Substrat: Referenzwerte in Abhängigkeit von Alter und Geschlecht unter Berücksichtigung hormonaler Einflüsse und Schwangerschaft
}

\author{
Von D. H. den Blaauwen
}

Dokter J. H. Jansenziekenhuis, Emmeloord, Niederlande

Stadsziekenhuis „De Engelenbergstichting“, Kampen, Niederlande

\author{
W. A. Poppe und W. Tritschler
}

Boehringer Mannheim GmbH, Diagnostica Forschung, Mannheim

(Eingegangen am 5. Juli/6. Dezember 1982)

Zusammenfassung: Für Cholinesterase mit Butyrylthiocholin-iodid als Substrat werden für $25^{\circ} \mathrm{C}$ Referenzwerte im Serum angegeben.

Bei Kindern (1-15 Jạhre, $N=309)$ wurden weder eine Altersabhängigkeit noch geschlechtsspezifische Unterschiede gefunden.

Bei Männern (16-94 Jahre, $N=718$ ) wurde ebenfalls keine Altersabhängigkeit gefunden; gegenüber dem Referenzbereich der Kinder ließ sich kein Unterschied in der Lage und Verteilung der katalytischen Konzentrationen an Cholinesterase nachweisen.

Bei Frauen (16-99 Jahre, $\mathrm{N}=861$ ) zeigte sich bei den Cholinesterase-Werten ein altersbedingter Unterschied:

Unabhängig von Schwangerschaft oder der Einnahme von hormonalen Kontrazeptiva werden in den jüngeren Lebensjahren (16-39 Jahre) niedrigere katalytische Konzentrationen gefunden als in späteren Lebensjahren ( $\geqslant 40$ Jahre); die Referenzwerte dieser Altersgruppe (40=99 Jahre) unterscheiden sich nicht von denen der Männer und Kinder. Wị schlagen deshalb folgende Referenzbereiche vor (jeweils 2,5. bis 97,5. Perzentil):

Kịnder, Männer sowie Frauen ab 40 Jahre:

Frauen (16-39 Jahre, nicht-sçhwanger, keine hormonalen Kontrazeptiva):

Frauen (18-41 Jahre, schwanger bzw. Einnahme von Kontrazeptiva):
3,5 bis $8,5 \mathrm{kU} / 1$

2,8 bis $7,4 \mathrm{kU} / \mathrm{l}$

2,4 bis $6,0 \mathrm{kU} / \mathrm{l}$

Cholinesterase (EC 3.1.1.8) with butyrylthiocholine iodide as substrate:

Age- and sex-dependent reference values with special reference to hormonal effects and pregnancy

Summary: Reference values are reported for the assay of cholinesterase (substrate: butyrylthiocholine iodide) in serum at $25^{\circ} \mathrm{C}$. There was no evidence of any age-dependency or sex-specific distinction in children ( 1 to 15 years, $N=309$ ), nor were there any age-dependent changes observed in males (16 to 94 years, $N=718$ ). Compared with the reference range for children, there was no detectable difference in the location and distribution of catalytic cholinesterase concentrations.

In females (16 to 99 years, $N=861$ ), an age-dependent difference in cholinesterase values became apparent:

Regardless of pregnancy or use of hormonal contraceptives, the catalytic concentrations were found to be lower in younger females ( 16 to 39 years) than in older ones ( $\geqslant 40$ years); the reference values in the older age

J. Clin. Chem. Clin. Biochem. / Vol. 21, 1983 / No. 6 
group (40 to 99 years) did not differ from those of males and children. Therefore the following reference values are proposed (in each case 2.5 to 97.5 percentile):

Children, males and females above 40 years:

3.5 to $8.5 \mathrm{kU} / \mathrm{l}$

Females (16-39 years, nonpregnant, not taking hormonal contraceptives):

2.8 to $7.4 \cdot \mathrm{kU} / \mathrm{l}$

Females (18-41 years, pregnant or taking hormonal contraceptives):

2.4 to $6.0 \mathrm{kU} / \mathrm{l}$

\section{Einführung}

Für die Bestimmung der katalytischen Konzentration der Cholinesterasen (Acylcholinacylhydrolase, EC 3.1.1.8) haben sich als Substrate Acetylthiocholin-iodid und Butyrylthiocholin-iodid durchgesetzt.

Für Acetylcholinesterasen liegen eingehende Untersuchungen vor zum Einfluß von Geschlecht, Alter und oralen Kontrazeptiva $(1,2,3)$ : Männer haben höhere katalytische Konzentrationen im Plasma oder Serum als Frauen. Bei Frauen, die hormonale Kontrazeptiva eihnehmen, findet man niedrigere Werte als bei Frauen, die keine hormonalen Kontrazeptiva einnehmen. Nach dem 60 . Lebensjahr werden bei Frauen erhöhte katalytische Konzentrationen gefunden. Geschlechtsspezifische Unterschiede sind ab diesem Alter nicht mehr nachweisbar. Bis zum 50. Lebensjahr soll bei Männern und Frauen keine Altersabhängigkeit bestehen. Man kann davon ausgehen, da $B$ in der Schwangerschaft die katalytische Konzentration der Acetylcholinesterasen häufig erniedrigt ist (3). Für Pseudocholinesterasen (Substrat: Butyrylthiocholin-iodid) sind ähnlich umfangreiche Untersuchungen nicht bekannt geworden. Jedoch sind auch hier für Männer höhere Referenzwerte als für Frauen mitgeteilt worden $(4,5)$.

Wir haben deshalb Referenzwerte bei Kindern und Erwachsenen ermittelt und sind den genannten Einflüssen auf die katalytische Konzentration der Cholinesterase nachgegangen.

\section{Material und Methoden \\ Reagenzien, Methoden, Geräte}

Test-Combination Cholinesterase (EC 3.1.1.8, Substrat: Butyrylthiocholin-iodid, Best.-Nr. 124 133) sowie Testpackungen zur Bestimmung von Alaninaminotransferase (EC 2.6.1.2) und $L-\gamma$ Glutamyltransferase (EC 2.3.2.2) entstammten dem Lieferprogramm von Boehringer Mannheim GmbH, D-6800 Mannheim 31.

Nach der Arbeitsanleitung des Herstellers wurde an einem Reaction Rate Photometer von Vitatron (Dieren, Niederlande) die Zeit $(t)$ in s für eine Absorptionsänderung von $\Delta A=0,100$ bei $405 \mathrm{~nm}$ gemessen. Gegenüber der Originalmethode (6) ist der verwendete Test in Bezug auf die Substratkonzentration optimiert.
Endkonzentrationen im Test: $7 \mathrm{mmol} / \mathrm{l}$ Butyrylthiocholin-iodid; $50 \mathrm{mmol} / \mathrm{l}$ Phosphat-Puffer, $\mathrm{pH}$ 7,7; 0,25 mmol/1 5,5'-Dithiobisnitrobenzoesäure.

Berechnung: katalytische Konzentration $\left(25^{\circ} \mathrm{C}\right)[\mathrm{U} / 1]=\frac{70376}{\mathrm{t}}$. Probanden

Das Referenzkollektiv der Kinder und Erwachsenen $(N \equiv 1888)$ umfaßt ambulante Patienten aus dem vorwiegend lạndwirtschaftlich orientierten Einzugsbereich der Städte Kampen und Emmeloord (Mittelholland). Mit einem Erhebungsbogen wurden erfaßt: Alter, Geschlecht, Schwangerschaft, Einnahme von Óvulationshemmern. Im Kollektiv nicht enthalten sind Patienten mit schweren Erkältungeñ und gripppalen Infekten. Zur Bíldung des Referenzkollektivs wurden Patienten mit alkoholgefährdeten Berufen, Berufen im Chemie-Bereich sowie Patienten, bei denen eine Kontaminierung mit Insektiziden und Pestiziden möglich war, ausgeschlossen.

Patienten mit Uber- oder Untergewicht (Abweichungen: mehr als $20 \%$ vom Normalgewicht) sind im Referenzkollektiv nicht enthalten. Auf eine generelle Medikamentenanamnese wurde ver: zichtet. Jedoch wurden von älteren Patienten ( $>60$ Jahrè) häựig Anàlgetikă, Kardiaka und Antihypertonika eingenommen.

Zur Auswahl des Referenzkollektivs wurden außerdem klinischchemische Kenngrößen eingesetzt, welche bei Lebeřerkranknungen verändert sind. Referenzwerte: Alañinaminotransferase $\left(37^{\circ} \mathrm{C}\right)$ : Männer bis $40 \mathrm{U} / \mathrm{l}$, Frauen bis $35 \mathrm{U} / \mathrm{l} ; \dot{L}-\gamma=$ Glutamyltransferase $\left(25^{\circ} \mathrm{C}\right)$ : Männer bis $28 \mathrm{U} / \mathrm{l}$, Frauen bis $18 \mathrm{U} / \mathrm{l}$.

Auf die Bestimmung der Dibucain-Zahl žur Erkennung von genetisch bedingtem Cholinesterase-Mangel haben wir verzichtet, weil dieser Stoffwechseldefekt relativ selten aúftritt (Häufigkeit etwa $1: 1500$ ).

\section{Probennahme und Qualitätssicherung}

Die Blutabnahme erfolgte vormittags. Nach Lösen der Stauung wurde Blut für die Serumgewinnung abgenommen. Serum wurde durch 10 inin Zentrifugation bei $2000 \mathrm{~g}$ erhalten und bei $-20^{\circ} \mathrm{C}$ eingefroren. Die katalytischen Konzentrationen der Alaninaminotransferase und der $L-\gamma$-Glutamyltíansferase wurden jeweils nach 3 Wochen bestimmt. Wenn die gemessenen Werte innerhalb des vorgegebenen Referenzbereiches lagen und die Anamnese die Kriterien des Referenzkollektivs erfüllte, wurde die katalytische Konzentration an Cholinesterase in Doppelbestimmungen untersucht. Die katalytische Konzentration der Cholinesterase bleibt innerhalb des angegebenen Zeitraumes unverändert (7). Die Präzision der Meßergebnisse sowie die Wiederfindung an Cholinesterase wurden mit den Kontrollseren Precilip (Chargen 763 und 764) und Hyland (Lot No. 2 and No. 4) ermittelt.

Präżision uñd Wièderfindung von Alaninaminotransferase und $L$ $\gamma$-Glutamyltransferase wurden mit anderen handelsüblichen Kontrollseren überprüft (Wiederfindung: $\pm 10 \%$ des Sollwertes; $\mathrm{VK}_{\text {Tag/Tag }}<\tilde{5 \%)}$.

\section{Statistische Methoden}

Die Präżisionen in der Serie und von Serie zu Serie wurden mit Hilfe der einfachen Varianzanalyse berechńet. 
Die Verteilung der Meßwerte wurde durch Angabe der Perzentile beschrieben. Da bei der Größe der vorliegenden Kollektive auch die Ränder der Verteilung der katalytischen Konzentrationen an Cholinesterase ausreichend gut erfaßt sind, haben wir uns entschieden, als Unter- bzw. Obergrenze das 2,5. bzw. 97,5. Perzentil anzugeben. Extreme Werte, die trotz der angewandten Ausschlußkriterien im Kollektiv enthalten sein könnten und die Grenzen des Referenzbereiches beeinflussen könnten, werden damit sicher ausgeschlossen. Zur Uberprüfung der Einflußgrößen Alter, Geschlecht und Ovulationshemmer wurde der U-Test von Mann-Whitney eingesetzt (8). Für Kinder wurde geprüft, ob sich in Lage und Varianz der katalytischen Konzentrationen an Cholinesterase für Mädchen und Jungen Unterschiede zeigen (UTest, Moses' rank like test, (9). Als Irrtumswahrscheinlichkeit wurde jeweils $\alpha=0,05$ festgelegt.

\section{Ergebnisse}

\section{Qualitätssicherung}

Tabelle 1 enthält die Ergebnisse der Qualitätssicherung von Cholinesterase in Kontrollseren. Die Präzision in der Serie und die Präzision von Tag zu Tag waren gut (VK: 2,0 bis 4,6\%). Systematische Abweichungen zu den angegebenen Sollwerten wurden nur bei einem Kontrollserum (Hyland lot No. 2) beobachtet. Da jedoch jeweils zwei Kontrollseren von verschiedenen Herstellern eingesetzt wurden, kann unterstellt werden, da $B$ sich die Analysenmethode während des gesamten Untersuchungszeitraumes unter Kontrolle befand.

\section{Altersverteilung des Referenzkollektivs}

Tabelle 2 zeigt die Zusammensetzung des Referenzkollektivs von 1888 Probanden. Die Besetzung der einzelnen Altersgruppen ist relativ homogen, die Gruppen sind mit ausreichend vielen Personen belegt.

\section{Referenzwerte bei Kindern}

In Abbildung 1 ist die Verteilung der katalytischen Konzentrationen an Cholinesterase für Jungen und Mädchen im Alter von 1 bis 15 Jahren dargestellt. Eine Altersabhängigkeit der Cholinesterase-Werte war statistisch nicht nachweisbar; ein Unterschied in Lage und Verteilung der Cholinesterase-Werte zwischen Jungen und Mädchen ließ sich statistisch ebenfalls nicht absichern.

Der 95\%-Bereich zwischen dem 2,5. und dem 97,5. Perzentil liegt bei Jungen zwischen 3,9 und $8,8 \mathrm{kU} / \mathrm{l}$, bei Mädchen zwischen 3,2 und 8,5 kU/1 (Tab. 3).

\section{Referenzwertè bei Männern}

In Abbildung 2 ist die Verteilung der katalytischen Konzentrationen an Cholinesterase für Männer (16-94 Jahre) wiedergegeben. Eine Altersabhängigkeit ist nicht erkennbar und statistisch nicht nachweisbar. Der 95\%-Bereich liegt zwischen 3,2 und $8,5 \mathrm{kU} / 1$ (Tab. 4).

Tab. 1. Cholinesterase: Wiederfindung und Präzision in Kontrollseren.

\begin{tabular}{|c|c|c|c|c|c|c|c|c|c|c|c|}
\hline \multirow[t]{3}{*}{ Kontrollserum } & \multirow{2}{*}{\multicolumn{2}{|c|}{$\begin{array}{l}\text { Herstellerangaben } \\
\text { Sollwert [kU/l] }\end{array}$}} & \multirow[t]{3}{*}{$\mathbf{N}$} & \multirow{2}{*}{\multicolumn{3}{|c|}{$\begin{array}{l}\text { Wiederfindung* } \\
\text { Cholinesterase [kU/1] }\end{array}$}} & \multirow[b]{3}{*}{$95 \%$} & \multirow[b]{3}{*}{ Max. } & \multicolumn{2}{|c|}{ Präzision** } & \multirow{3}{*}{$\begin{array}{l}\mathrm{VK}_{\mathrm{T} / \mathrm{T}} \\
{[\%]}\end{array}$} \\
\hline & & & & & & & & & & $\mathrm{VK}_{\mathbf{S}}$ & \\
\hline & $\overline{\mathbf{x}}$ & Bereich & & Min. & $5 \%$ & $50 \%$ & & & {$[\mathrm{kU} / \mathrm{ll}]$} & {$[\%]$} & \\
\hline Precilip ${ }^{\otimes}$ Charge 763 & 3,30 & $2,90-3,70$ & 85 & 3,13 & 3,20 & 3,35 & 3,61 & 3,70 & 3,36 & 2,4 & 4,4 \\
\hline Precilip Charge 764 & 3,34 & $2,80-3,87$ & 12 & 3,20 & 3,20 & 3,28 & 3,44 & 3,44 & 3,28 & 2,5 & 2,8 \\
\hline Hyland Lot No. 2 & 3,26 & $2,95-3,57$ & 44 & 3,28 & 3,35 & 3,61 & 3,91 & 3,91 & 3,62 & 2,0 & 4,6 \\
\hline Hyland Lot No. 4 & 3,06 & $2,62-3,50$ & 57 & 2,82 & 2,87 & 3,00 & 3,13 & 3,21 & 3,01 & 2,4 & 3,3 \\
\hline
\end{tabular}

* Die Werte gèben dạs 5., 50. (Median), 95. Perzentil sowie niedrigste und höchste Mittelwerte aus N Doppelbestimmungen an für 30 MeBtage. Bei einer Serie von 20 Patientenseren wurden 2 Kontrollseren mitgeführt: Precilip ${ }^{\circledR}$ nach 10 Seren und Hyland nach 10 weiteren Seren.

** Die Präzision in der Serie bzw. von Tag zu Tag wurḍe auṣ N Doppelbestimmungen an 30 Meßtagen berechnet.

Tab. 2. Altersvertẹilung des Referenzkollèktivs $(\mathrm{N}=1888)$.

\begin{tabular}{|c|c|c|c|c|c|c|c|c|c|c|}
\hline & \multicolumn{2}{|c|}{ Alter [Jahre] } & \multirow[b]{2}{*}{$20-29$} & \multirow[b]{2}{*}{$30-39$} & \multirow[b]{2}{*}{$40-49$} & \multirow[b]{2}{*}{$50-59$} & \multirow[b]{2}{*}{$60-69$} & \multirow[b]{2}{*}{$70-79$} & \multirow[b]{2}{*}{$80-99$} & \multirow[t]{2}{*}{ Summe } \\
\hline & $1-9$ & $10-19$ & & & & & & & & \\
\hline Jungen und Männer & 103 & 105 & 102 & 103 & 100 & 100 & 106 & 101 & 53 & 873 \\
\hline $\begin{array}{l}\text { Mädchen und Frauen ohne } \\
\text { hormonale Kontrazeption }\end{array}$ & 104 & 97 & 50 & 50 & 91 & 104 & 101 & 109 & 68 & 774 \\
\hline $\begin{array}{l}\text { Frauen mit hormonaler } \\
\text { Kontrazeption }\end{array}$ & & & 54 & 25 & & & & & & 79 \\
\hline Schwangere & & 3 & 96 & 62 & 1 & & & & & 162 \\
\hline
\end{tabular}




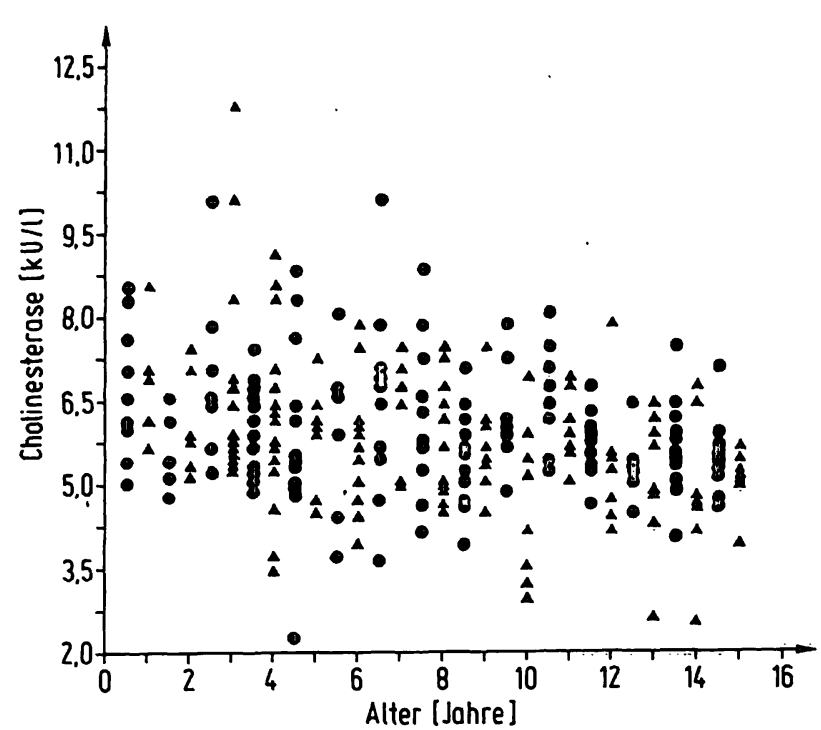

Abb. 1. Häufigkeitsverteilung der katalytischen Konzentrationen an Cholinesterase im Serum von Kindern $(N=309)$ in Abhängigkeit vom Alter (1 bis $15 \mathrm{Jahre}$ ).

Wegen der besseren Ubersichtlichkeit wurden die katalytischen Konzzentrationen bei Jungen gegenüber den Werten bei Mädchen um ein halbes Jahr versetżt eingezeichnet.

O Jungen $(\mathrm{N}=155) \quad \Delta$ Mädchen $(\mathrm{N}=154)$

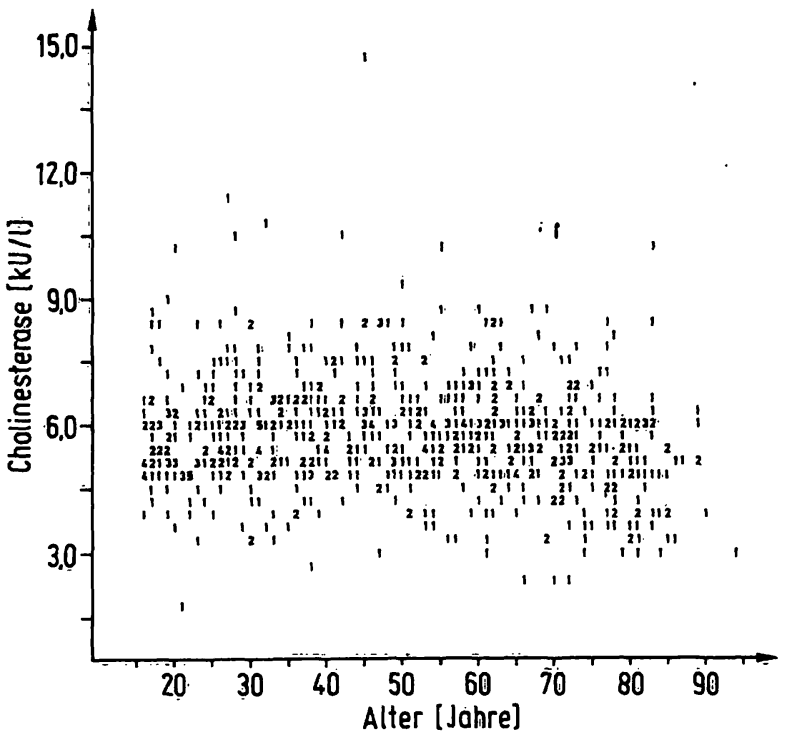

Abb. 2. Häufigkeitsverteilung der katalytischen Konzentrationen an Cholinesterase im Serum der Männer $(N=715)$ in Abhängigkeit vom Alter (16 bis 94 Jahre $)$.

Tab. 3. Verteilung der katalytischen Konzentrationen an Cholinesterase bei Kindern.

Die Werte geben das 2,5., 5., 50 (Median), 95. und 97,5. Perzentil sowie niedrigste und höchste Meßwerte fürr N Kinder im Alter von $1 \mathrm{Jahr}$ bis $15 \mathrm{Jahren}$ an.

Prüfung auf Unterschiede zwischen Jungen und Mädchen (U-Test bżw. Moses' rank like test, $\alpha=0,05$ ): këinē Signifikanz.

\begin{tabular}{lllcccrrr}
\hline & N & \multicolumn{3}{c}{ Cholinesterase $[\mathrm{kU} / \mathrm{l}]$} & & & & \\
& & Min. & $2,5 \%$ & $5 \%$ & $50 \%$ & $95 \%$ & $97,5 \%$ & $\mathbf{3} \%$ \\
\hline Jungen & 155 & 2,27 & 3,91 & 4,47 & 5,87 & 8,04 & 8,80 & 10,05 \\
Mädchen & 154 & 2,51 & 3,20 & 3,91 & 5,63 & 7,82 & 8,53 & 11,73 \\
Kinder & 309 & 2,27 & 3,61 & 4,14 & 5,75 & 8,04 & 8,53 & 11,73 \\
\hline
\end{tabular}

Tab. 4. Verteilung der katalytischen Konzentrationen an Cholinesterase bei Männern und Frauen.

Die Werte geben das 2,5., 5., 50. (Median), 95. und 97,5. Perzentil sowie niedrigste und höchste Meßwerte für N Männer und Frauen an.

Prüfung auf Unterschiede zwischen den Gruppen (U-Test, $\alpha=0,05$ ):

Männer - Frauen (ohne hormonale Kontrazeption, 16 bis 39 Jahre):

Frauen ohne hormonale Kontrazeption:

Altersgruppe 16 bis 39 Jahre - Altersgruppe 40 bis 99 Jahre:

Frauen (mit hormonaler Kontrazeption) - Schwangere:

Signifikanz

Signifikanz

keine Signifikanz

\begin{tabular}{|c|c|c|c|c|c|c|c|c|c|}
\hline & \multirow{2}{*}{$\begin{array}{l}\text { Alter } \\
\text { [Jahre] }\end{array}$} & \multirow[t]{2}{*}{$\mathbf{N}$} & \multicolumn{7}{|c|}{ Cholinesterase [kU/l] } \\
\hline & & & Min. & $2,5 \%$ & $5 \%$ & $50 \%$ & $95 \%$ & $97,5 \%$ & Max. \\
\hline Männer & $16-94$ & 715 & 1,74 & 3,20 & 3,70 & 5,63 & 8,28 & 8,53 & 14,82 \\
\hline Frauen: & $16-39$ & 147 & 2,20 & 2,82 & 2,93 & 4,69 & 7,04 & 7,41 & 9,38 \\
\hline ohne hormonale Kontrazeption & $40-99$ & 473 & 1,42 & 3,52 & 3,70 & 5,41 & 7,82 & 8,80 & 20,11 \\
\hline mit hormonaler Kontrazeption & $20-39$ & 79 & 2,35 & 2,43 & 2,71 & 3,91 & 5,41 & 5,86 & 5,86 \\
\hline Schwangere & $18-41$ & 162 & 1,58 & 2,35 & 2,43 & 3,70 & 5,86 & 6,55 & 7,61 \\
\hline
\end{tabular}




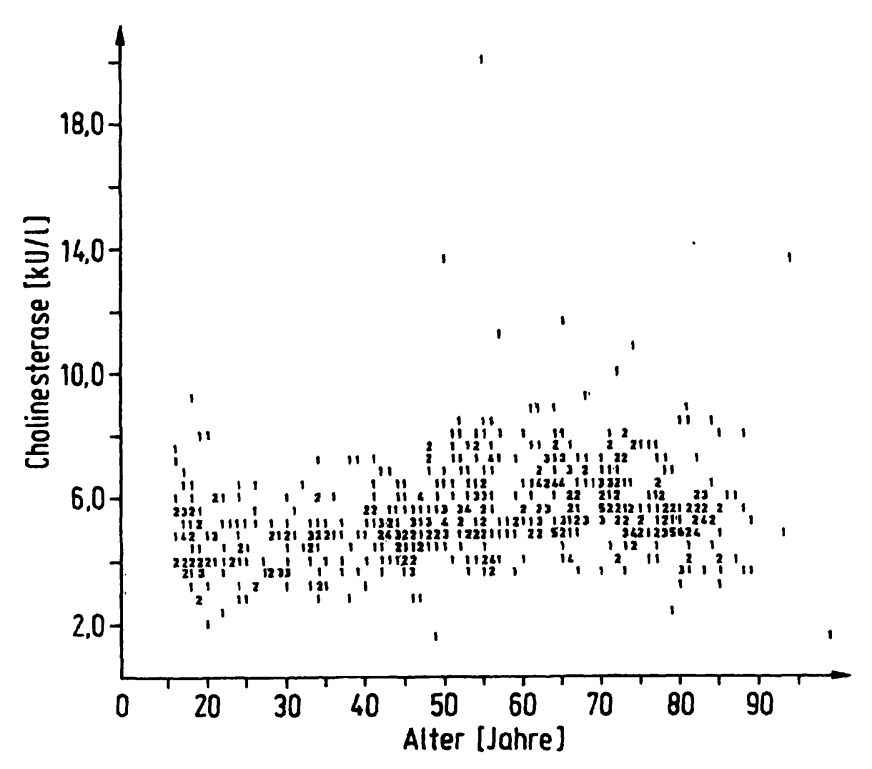

Abb. 3. Häufigkeitsverteilung der katalytischen Konzentrationen an Cholinesterase im Serum der Frauen (ohne hormonale Kontrazeptiva $(N=620)$ in Abhängigkeit vom Alter (16 bis 99 Jahre).

\section{Referenzwerte bei Frauen}

Abbildung 3 zeigt die Verteilung der katalytischen Konzentrationen an Cholinesterase für Frauen (16-99 Jahre, ohne hormonale Kontrazeption). Bis etwa 39 Jahre werden häufig niedrigere Werte als oberhalb dieser Altersgrenze gemessen. Die katalytischen Konzentrationen in den Altersgruppen 16-39 Jahre bzw. 40-99 Jahre zeigen einen Unterschied in der Lage, der sich statistisch nachweisen läßt. Dieser Unterschied ist jedoch erst im Rahmen der Daten-Analyse aufgefallen; das Testergebnis sollte deshalb nur zur Unterstützung einer entsprechenden Arbeitshypothese herangezogen werden. Der $95 \%$-Bereich reicht von 2,8 bis $7,4 \mathrm{kU} / 1$ (16-39 Jahre) bzw. 3,5 bis $8,8 \mathrm{kU} / 1$ (40-99 Jahre) (Tab. 4).

Frauen, die hormonale Kontrazeptiva einnehmen, sowie Schwangere haben niedrigere, jedoch miteinander vergleichbare $95 \%$-Bereiche: 2,4 bis $5,9 \mathrm{kU} / 1$ bzw. 2,4-6,6 kU/1. Die Prüfung auf Unterschiede der Cholinesterase-Werte zwischen diesen beiden Kollektiven ergab keine Sịnifikikanz.

\section{Diskussion}

Die diagnostische Bedeutung der Cholinesterase liegt darin, daß ihr Verhalten als „Funktions- und Leistungsparameter der Leber" von demjenigen der Aminotransferasen unabhängig ist und die Schwere einer akuten und das Stadium einer chronischen Lebererkrankung charakterisiert (10). Der Untergren- ze des. Referenzbereiches kommt damit eine besondere Bedeutung zu. Erhöhte katalytische Konzentrationen wurden besonders beim nephrotischen Syndrom gefunden (10).

Vergleicht man die bei Jungen und Männern gefundenen unteren Grenzen des $95 \%$-Bereiches miteinander $(3,9 \mathrm{kU} / 1$ bzw. $3,2 \mathrm{kU} / \mathrm{l})$, so kann eine gemeinsame Untergrenze diskutiert werden. Für beide Kollektive besteht keine Altersabhängigkeit der katalytischen Konzentrationen an. Cholinesterase, die Mediane als stabilste Lagekenngrößen unterscheiden sich praktisch nicht voneinander. Wir schlagen deshalb als gemeinsame Untergrenze des Referenzbereiches 3,5 kU/1, als Obergrenze 8,5 kU/1 vor. Für Mädchen (1 Jahr-15 Jahre) und Frauen, die älter als 40 Jahre sind, läßt sich ebenfalls eine Altersabhängigkeit der katalytischen Konzentrationen an Cholinesterase nicht nachweisen. Die 95\%-Bereiche beider Kollektive stimmen gut überein $(3,2$ bis 8,5 $\mathrm{kU} / 1$ bzw. 3,5 bis $8,8 \mathrm{kU} / \mathrm{l}$ ), so daß wir einen gemeinsamen Referenzbereich festlegen möchten: 3,5 bis $8,5 \mathrm{kU} / \mathrm{l}$.

Damit ergeben sich für Kinder sowie auch Männer und Frauen über 40 Jahre dieselben Referenzwerte: 3,5 bis $8,5 \mathrm{kU} / \mathrm{l}$.

Frauen im gebärfähigen Alter (16-39 Jahre) zeigen, unabhängig davon, ob hormonelle Einflüsse durch Kontrazeptiva oder Schwangerschaft vorliegen, niedrigere katalytische Konzentrationen an Cholinesterase. Für Frauen, die nicht schwanger sind und keine hormonalen Kontrazeptiva einnehmen, schlagen wir einen Referenzbereich von 2,8 bis $7,4 \mathrm{kU} / \mathrm{l}$ vor; für Schwangere bzw. Frauen, welche Kontrazeptiva einnehmen, ergibt sich ein Referenzbereich von 2,4 bis $6,0 \mathrm{kU} / \mathrm{l}$.

Unsere Ergebnisse zeigen, da $\mathrm{B}$ in den bisherigen $\mathrm{Re}$ ferenzwertuntersuchungen für Pseudocholinesterasen Aspekte nicht berücksichtigt wurden, die für Acetylcholinesterasen teilweise bekannt waren. Eine nicht erfolgte Differenzierung der katalytischen Konzentrationen an Cholinesterase bei Frauen bzw. die Nichteinbeziehung von Schwangerschaft und oralen Kontrazeptiva muß deshalb $\mathrm{zu}$ undifferenzierten Schlußfolgerungen führen. Möglicherweise erklären sich so die Literaturangaben, daß Männer höhere katalytische Konzentrationen an Cholinesterase haben als Frauen $(4,5)$.

\section{Danksagung}

Für die sorgfältige Durchführung der Untersuchungen danken wir J. van Dijk, W. J. Pelleboer-Troost, J. Boonemmer-de Jong und $C$. A. Quartel. 


\section{Literatur}

1. Sidell, F. R. \& Kaminskis, A. (1975) Clin. Chem. 21, 1393-1395.

2. Robertson, G. S. (1967) Lancet $I, 232-235$.

3. Redderson, C. L. (1973) Int. J. Clin. Pharmacol. 8, 51-57.

4. Prellwitz, W., Kapp, S. \& Müller, D. (1976) J. Clin. Chem. Clin. Biochem. 14, 93-97.

5. Szasz, G. (1968) Clin. Chim. Acta 19, 191-204.

6. Knedel, M. \& Böttger, R. (1967) Klin. Wochenschr. 45, 325-327.

7. Schwartz, M. K. (1973) Adv. Clin. Chem. 16, 10.

8. Conover, W. J. (1971) Practical nonparametrịc statistics J. Wiley \& Sons, New York.

9. Hollander, M. \& Wolfe, D. A. (1973). Nonparametric statistical methods J. Wiley \& Sons, New York.

10. Adolph, L. (1979) Münch. Med., , Wochenschr. 12̄1, $1527-1530$

Dr. W. Tritschler

Boehringer Mannheim GmbH

Erprobung Diagnostica

Sandhofer Straße 116

D-6800 Mannhieim 31

Drs. D. H. den Blaauwen, Klin. Chemiker Dokter J: H. Jansenziekenhuis

NL-8300 GA Emmeloord (Niederlande) und

Stadsziekenhuis „De Engelenbergstichting“ NL-8266 AB Kampen (Niederlande) 